\title{
HRTEM and EELS Study in GaN-based Diluted Magnetic Semiconductor
}

\author{
R. T. Huang*, C. F. Hsu*, J. J. Kai*, F. R. Chen* and T. S. Chin** \\ *Department of Engineering and system Science, National Tsing-Hua University, Hsinchu 300, \\ Taiwan, R. O. C. \\ **Department of Materials Science and Engineering, National Tsing-Hua University, Hsinchu 300, \\ Taiwan, R. O. C.
}

Diluted magnetic semiconductors (DMSs) based on III-V semiconductors, fabricated by incorporating a proper concentration of magnetic ions, have attracted considerable attentions in recent years because of the possibility involving charge and spin degrees of freedom in a single substance. It is expected to provide new functionality for microelectronic devices by exploiting the spin of charge carriers in the ferromagnetic semiconductors [1]. Also, it is desirable to exhibit robust ferromagnetism with Curie temperature Tc above room temperature without second magnetic phase or clusters [1]. However, to understand the correlation of magnetic properties and microstructure in DMSs with spatial resolution of near or even better than nanometer scale is required. The high spatial resolution available on a transmission electron microscopy (TEM) combined with the benefits of electron energy loss spectroscopy (EELS) allows detailed analysis in terms of microstructure and the white-line intensities of the transition metal at the onsets of the $L_{2}$ and $L_{3}$ absorption edges on the scale of nanometer. In our experiment, GaN-based DMSs with several annealing conditions are investigated by using high resolution TEM coupling with nano-beam energy dispersive x-ray spectroscopy (EDX) and EELS.

In this study, for avoiding the formation of second phase (as shown in Fig. 1), we report on the use of a thin protecting layer during implantation with $\mathrm{Ni}$ at a dose of $5 \times 10^{16} \mathrm{~cm}^{-2}$ and the study of corresponding magnetic and structural properties after subsequent annealing. After etching off the protecting layer, subsequent annealing up to $800{ }^{\circ} \mathrm{C}$ under flowing $\mathrm{N}_{2}$ resulted in a p-type GaN with apparent ferromagnetic behavior up to $\sim 320 \mathrm{~K}$. In addition, the ferromagnetic behavior became more manifest with increasing annealing temperature that increases hole concentration. Neither presence of any other second phases nor clusters in the Ni-implanted region was identifiable, at least to the 0.2 $\mathrm{nm}$ point-to-point resolution of high resolution transmission electron microscopy. Although implantation damages are still abundant, there are no obvious extra spots contributed from secondary phases, and only the streaking diffraction spots from the GaN hexagonal crystal structure is observed. The streaking diffraction spots are resultant from lattice defects caused by the damages of ion implantation, including dislocation loops or stacking faults, as shown in Fig. 2. Since the 3- $d$ shell is the outermost shell and known to be responsible for the magnetic properties for the 3-d transition metal group ions [2], this work will study the behavior of $3 d$ state electrons in Ni-doped DMSs. Figure 2 shows the electron energy loss spectrum from several nanowires. The characteristic nickel $L_{2}$ and $L_{3}$ absorption edge can be seen. Further calculations on the ratio of the integrated intensity counts, done on the $L_{3}$ and $L_{2}$ absorption edge of nickel, correspond to valence state of +2 . In analogy with Wahl et al.'s report for Fe implanted GaN [3], we expect that some fraction of Ga is substituted by $\mathrm{Ni}$ after implantation. Moreover, the post-implantation annealing assured the migration to the correct (cation) lattice site of most of the implanted dopant ions [4]. With increasing annealing temperature, more implanted $\mathrm{Ni}$ ions migrate to the cation lattice sites, resulting in higher hole concentration, and consequently the more mediated ferromagnetic property. Consequently, this novel 
indirect implantation process that being easy to implement appears promising for attaining room temperature diluted magnetic semiconductors which are applicable to magneto-transport, magneto-optical and spintronics devices, among others.

\section{Reference}

1. T. Dietl, H. Ohno, F. Matsukura, J. Cibert, and D. Ferrand, Science, 287 (2000) 1019

2. C. Kittel, Introduction to Solid State Physic, Wiley, New York, 1996, p. 426

3. U. Wahl, A. Vantomme, G. Langouche, J. G. Correia, L. Peralta, and the ISOLDE collaboration, Appl. Phys. Lett., 78 (2001) 3217

4. A. F. Hebrd, R. P. Rairigh, J. G. Kelly, S. J. Pearton, C. R. Abernathy, S. N. G. Chu, and R. G. Wilson, J. Phys. D: Appl. Phys., 37 (2004) 511

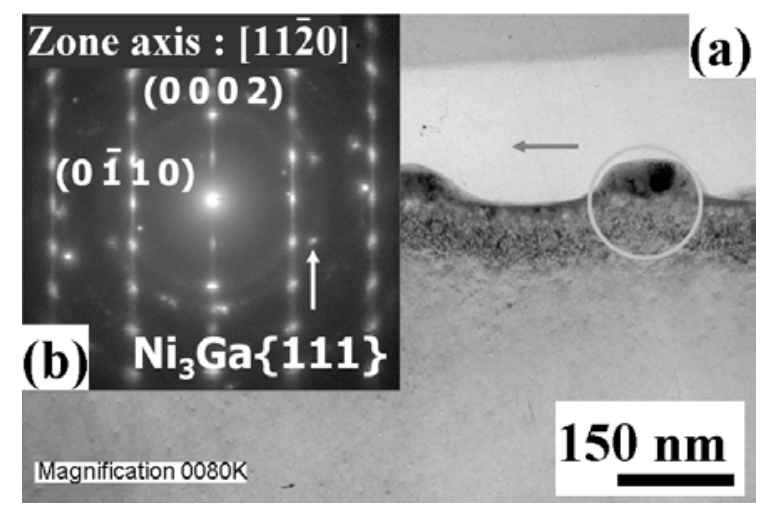

Fig. 1 The cross-sectional TEM image of a 5 at.\% Ni-implanted $\mathrm{GaN}$ film without the $\mathrm{Ni}$ protecting layer during ion implantation. It shows the $\mathrm{Ni}_{3} \mathrm{Ga}$ second phase.

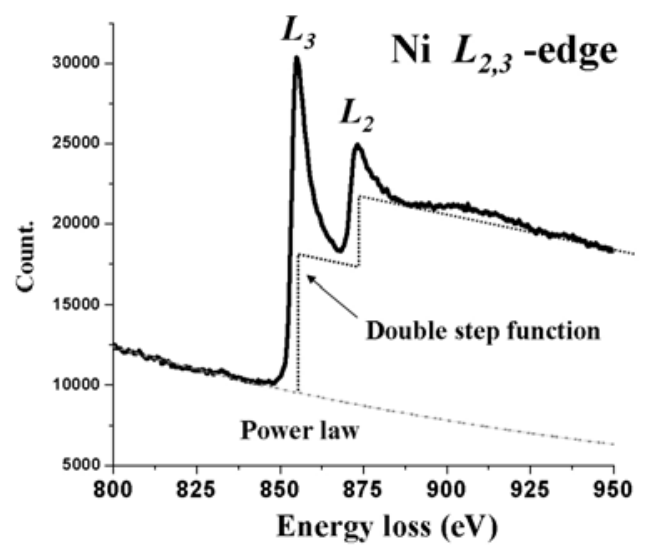

Fig. 3. The EELS spectrum of a 5 at.\% Ni-implanted GaN film after annealing at 800 ${ }^{\circ} \mathrm{C}$ for $5 \mathrm{~min}$.
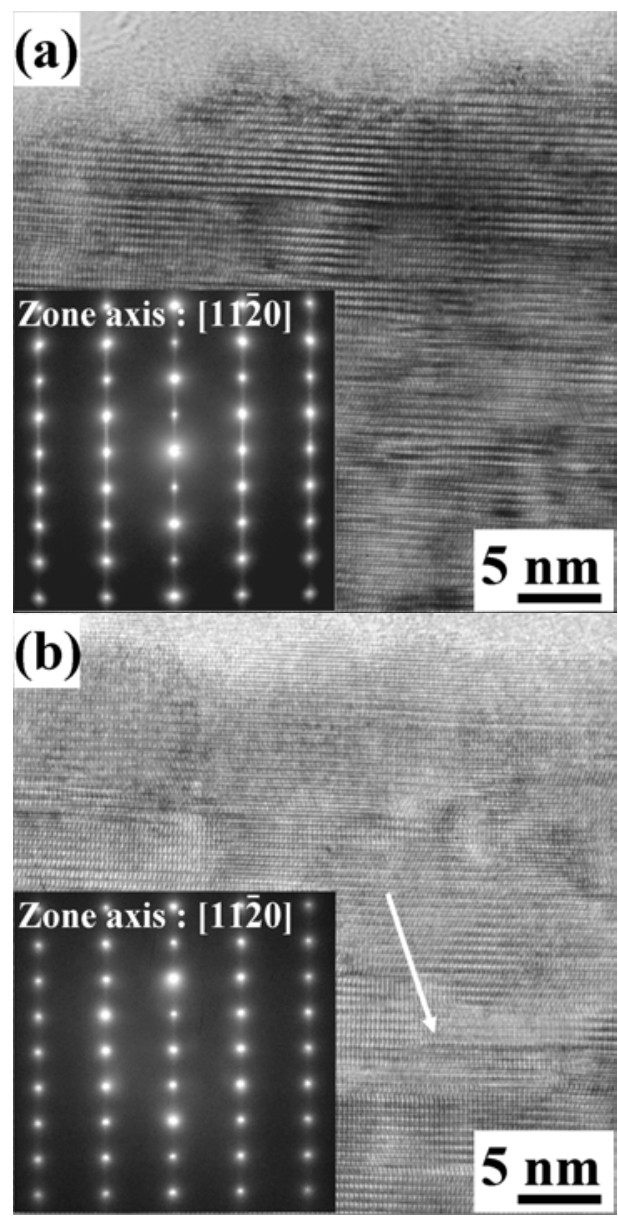

Fig. 2 The cross-sectional HRTEM image and SAD analysis of a 5 at.\% Ni-implanted GaN with the $\mathrm{Ni}$ protecting layer during ion implantation, (a) as-implanted and (b) post-annealing $\left(800^{\circ} \mathrm{C}\right.$ for $\left.5 \mathrm{~min}\right)$ 